\title{
Collateral damage: Impact of SARS-CoV-2 pandemic in people living with HIV
}

\author{
Sarah A. Cooley ${ }^{1}$ (D) $\cdot$ Brittany Nelson $^{1} \cdot$ John $^{\text {Doyle }}{ }^{1} \cdot$ Alexander Rosenow $^{1} \cdot$ Beau M. Ances $^{1,2,3}$
}

Received: 24 July 2020 / Revised: 2 November 2020 / Accepted: 17 November 2020 / Published online: 6 January 2021

(c) Journal of NeuroVirology, Inc. 2021

\begin{abstract}
People living with HIV (PLWH) may be at higher risk for adverse outcomes indirectly associated with the severe acute respiratory syndrome coronavirus (SARS-CoV-2). When comparing responses to questionnaires administered when social distancing and quarantine guidelines were first implemented, we found that PLWH were more likely to have restricted access to medical care, increased financial stress, increased symptoms of anxiety and depression, and increased substance use compared to demographically-similar people without HIV.
\end{abstract}

Keywords HIV · COVID-19 · Depression · Substance use

As of October 2020, over 8 million people in the United States have been diagnosed with severe acute respiratory syndrome coronavirus 2 (SARS-CoV-2) (CDC COVID Data Tracker, https://covid.cdc.gov/covid-data-tracker/). While there is currently little evidence to suggest people living with HIV (PLWH) are more susceptible to acquiring SARS-CoV-2 infection compared to the general population, PLWH may be at greater risk for adverse outcomes indirectly associated with the coronavirus (Inciarte et al. 2020; Park et al. 2020).

Here, we describe the effects of factors secondary to SARS-CoV-2, including quarantine and social isolation that was initially implemented and their effects on access to medical care, financial burden, mental health and substance use in PLWH and demographicallysimilar persons without HIV (HIV-) who have not been diagnosed with SARS-CoV-2. In April and May 2020 during the early onset of the pandemic, questionnaires were administered through telephone or email to PLWH (mean age $=50.3, \mathrm{SD}=12.7$; age range $=26-79$ ) and HIV- $($ mean age $=48.5, \mathrm{SD}=14.1$; age range $=25-74$ )

Sarah A. Cooley

scooley22@wustl.edu

1 Department of Neurology, Washington University in Saint Louis, Saint Louis, MO 63110, USA

2 Department of Radiology, Washington University in Saint Louis, Saint Louis, MO 63110, USA

3 Hope Center for Neurological Disorders, Washington University in Saint Louis, Saint Louis, MO 63110, USA who previously participated (within the past 3 years) in studies at Washington University in St. Louis. All participants gave written informed consent and were compensated for their participation.

Our PLWH participants represent a primarily older, disadvantaged group as indicated by the area deprivation index $\left(77^{\text {th }}\right.$ percentile) (Kind and Buckingham 2018). PLWH perceived significantly decreased access to medical care and greater financial stress, compared to HIV- ( $p$-values $<0.05$ ) despite no difference in the rate of job or income loss (Table 1). There was a trend-level relationship for food insecurity, with a greater percentage of PLWH indicating that they have recently gone hungry due to not having enough food. Additionally, PLWH indicated significantly more depressive symptoms, anxiety symptoms, and feelings of loneliness compared to HIV-. Anxiety levels had increased more from a previous visit prior to the pandemic with regards to the current survey for PLWH compared to HIV-. Use of tobacco and marijuana in the previous 30 days, and change in marijuana use from the previous study visit, was significantly greater in PLWH compared to HIV-. Greater marijuana use was significantly correlated with higher symptoms of depression $(r=0.25$, $p=0.008)$ and anxiety $(\mathrm{r}=0.22, p=0.01)$.

These data suggest a potential significant effect of the SARS-CoV-2 pandemic on the well-being of individuals even in the absence of a positive diagnosis. These effects may be greater in populations that are more marginalized, such as PLWH. It may be beneficial to further explore 
Table 1 Demographic characteristics and questionnaire data

\begin{tabular}{|c|c|c|c|}
\hline Measure & HIV- $(n=54)$ & PLWH $(n=133)$ & $p$-value \\
\hline Months since previous study visit; M (SD) & $20.8(15.6)$ & $19.4(12.3)$ & \\
\hline \multicolumn{4}{|l|}{ Demographics } \\
\hline Age; M(SD) & $48.5(14.1)$ & $50.3(12.7)$ & 0.46 \\
\hline Sex (\% Male) & $54 \%$ & $67 \%$ & 0.09 \\
\hline Race (\% AA) & $56 \%$ & $68 \%$ & 0.12 \\
\hline Years of education; M(SD) & $14.1(2.1)$ & $13.3(2.5)$ & 0.1 \\
\hline National Area Deprivation Index; M(SD) & $68.6(14.0)$ & $77.2(16.6)$ & $\mathbf{0 . 0 3}$ \\
\hline \multicolumn{4}{|l|}{ Clinical Characteristics of PLWH } \\
\hline Nadir CD4 T-cell count, Median (IQR) & - & $198(87-264)$ & - \\
\hline Recent CD4 T-cell count, Median (IQR) & - & $620(493-847)$ & - \\
\hline Undetectable viral load $(\%<50$ copies $/ \mathrm{mL})$ & - & $96 \%$ & - \\
\hline \multicolumn{4}{|l|}{ Medical Access and Financial Burden } \\
\hline $\begin{array}{l}\text { "Since March 1, 2020, I have limited access/lost access to medical care; or, the way my medical care } \\
\text { has been delivered has changed and does not meet my needs as well as before" (\% Yes) }\end{array}$ & $3.7 \%$ & $13.5 \%$ & $<.001$ \\
\hline $\begin{array}{l}\text { Current financial stress scale rating (On a scale of } 0 \text { (no financial stress) to } 10 \text { (severe financial } \\
\text { stress)); } \mathrm{M}(\mathrm{SD})\end{array}$ & $3.2(2.3)$ & $5.4(2.7)$ & 0.001 \\
\hline Change in financial stress scale rating from January $2020 ; \mathrm{M}(\mathrm{SD})$ & $+0.9(1.7)$ & $+2.1(2.6)$ & $\mathbf{0 . 0 3}$ \\
\hline $\begin{array}{l}\text { "In the past month, was there any day when you or anyone in your family went hungry because you } \\
\text { did not have enough food?" (\% Yes) }\end{array}$ & $2 \%$ & $10 \%$ & 0.09 \\
\hline $\begin{array}{l}\text { "Since the start of the COVID pandemic in the US, have you taken a cut in wage, salary, self- } \\
\text { employed income, or involuntary lost a job? (\% Yes) }\end{array}$ & $47 \%$ & $38 \%$ & 0.33 \\
\hline \multicolumn{4}{|l|}{ Mental Health } \\
\hline Beck Depression Inventory-II (BDI-II) Score; M(SD) & $7.8(7.1)$ & $13.8(11.4)$ & 0.009 \\
\hline Change in BDI-II Score since previous study visit; M(SD) & $+4.0(6.3)$ & $+5.0(6.7)$ & 0.51 \\
\hline Hospital Anxiety and Depression Scale (HADS)—Anxiety Subscale Score; M(SD) & $4.6(2.5)$ & $7.7(4.4)$ & 0.001 \\
\hline Change in HADS- Anxiety subscale score since previous visit; $\mathrm{M}(\mathrm{SD})$ & $+0.5(1.8)$ & $+2.1(2.3)$ & 0.04 \\
\hline UCLA Loneliness Scale & $14.1(10.4)$ & $23.0(13.9)$ & 0.003 \\
\hline \multicolumn{4}{|l|}{ Substance Use-30 Day KMSK } \\
\hline Alcohol subscale score & $3.3(3.6)$ & $3.8(3.9)$ & 0.43 \\
\hline Change in alcohol subscale score since previous visit & $+0.8(4.3)$ & $+1.0(3.3)$ & 0.85 \\
\hline Tobacco subscale score & $1.2(3.1)$ & $2.7(4.2)$ & 0.03 \\
\hline Change in tobacco subscale score since previous visit & $+0.4(2.9)$ & $+1.1(3.1)$ & 0.44 \\
\hline Marijuana subscale score & $0.6(2.1)$ & $3.0(4.3)$ & $<.001$ \\
\hline Change in marijuana subscale score since previous visit & $+0.1(0.3)$ & $+2.3(3.9)$ & 0.03 \\
\hline
\end{tabular}

Bolded values indicate significance at

$M$ mean, $S D$ standard deviation, $A A$ African American, IQR Interquartile range, KMSK Kreek-McHugh-Schluger-Kellogg scale $\mathrm{p}<.05$

the effects of SARS-CoV-2 in PLWH from a syndemic perspective, or the synergistic interaction of two or more epidemics producing increased disease burden in a specific population (Shiau et al. 2020). PLWH, particularly those already at greater risk for poorer mental health and substance abuse, could benefit from services and resources designed to help disadvantaged populations amidst the SARS-CoV-2 pandemic.
Acknowledgements The study was supported by grants from the National Institute for Nursing Research (R01NR015738) and the National Institute of Mental Health (R01MH118031).

\section{Reference}

Centers for Disease Control and Prevention, COVID-19 Response: COVID Data Tracker (2020) Retrieved October 23, 2020, from https://covid.cdc.gov/covid-data-tracker/ 
Inciarte A, Gonzalez-Cordon A, Rojas J, Torres B, de Lazzari E, de la Mora L, Martinez-Rebollar M, Laguno M, Callau P, Gonzalez-Navarro A, Leal L, Garcia F, Mallolas J, Mosquera M, Marcos MA, Ambrosioni J, Miro JM, Martinez E, Blanco JL (on behalf the COVID-19 in HIV Investigators) et al (2020) Clinical characteristics, risk factors, and incidence of symptomatic coronavirus disease 2019 in a large cohort of adults living with HIV: a single-center, prospective observational study AIDS: Oct 1, 34(12):1775-1780

Kind AJ, Buckingham WR (2018) Making neighborhood-disadvantage metrics accessible- The neighborhood atlas. N Engl J Med 378(26):2456-2458
Park LS, Rentsch CT, Sigel K et al (2020) COVID-19 in the largest US HIV cohort. AIDS 2020: 23rd International AIDS Conference Virtual. July 6-10 Abstract LBPEC23

Shiau S, Krause KD, Valera P, Swaminathan S, Halkitis PN (2020) The burden of COVID-19 in people living with HIV: a syndemic perspective. AIDS and Behavior, 24(8):2244-2249

Publisher's Note Springer Nature remains neutral with regard to jurisdictional claims in published maps and institutional affiliations. 